\title{
PROPERTY $P_{3}$ AND THE UNION OF TWO CONVEX SETS ${ }^{1}$
}

\section{E. O. BUCHMAN}

AbSTRACT. A set $S$ in a linear space is said to have the threepoint convexity property $P_{3}$ iff for each triple of points $x, y, z$ of $S$, at least one of the segments $x y, x z, y z$ is a subset of $S$. It is proved that if $S$ is a compact set in Euclidean space of dimension at least three with at least one point interior to its convex kernel and if the set of points of local nonconvexity of $S$ is interior to its convex hull, then $S$ has property $P_{3}$ iff it is the union of two convex sets.

Introduction. Property $P_{3}$ has been defined and investigated by Valentine for two-dimensional sets [3] and in finite-dimensional spaces [5]. A set $S$ in a linear space is said to have the three-point convexity property $P_{3}$ iff for each triple of points $x, y, z \in S$, at least one of the segments $x y, x z, y z$ is a subset of $S$. Although every set which is the union of two convex sets has property $P_{3}$, property $P_{3}$ alone does not characterize such sets, as the example of a five-pointed star shows. Valentine [3] has shown that in $E_{2}$ a closed set having property $P_{3}$ can be expressed as the union of three or fewer convex sets, and that the number three is best in this case. It is shown in this paper that under certain conditions a set in Euclidean space of dimension three (or higher) is the union of two convex sets if and only if it has property $P_{3}$. McKinney [2] has shown that if $S$ is a closed set in a topological linear space, then $S$ is the union of two convex sets if and only if it has the property that for any cyclically ordered $n$-tuple ( $n$ odd) of points of $S$, at least one of the segments connecting consecutive points is a subset of $S$. (This property implies property $P_{3}$.) Marr and Stamey [1] also have considered a property stronger than property $P_{3}$ which implies that $S$ is the union of two convex sets.

Preliminary definitions and results. A point $x$ of a set $S$ in $E_{n}$ is called a point of local nonconvexity of $S$ if for every neighborhood $N$ of $x$ there exists a pair of points $u, v \in S \cap N$ such that the segment $u v$ is not a subset of $S$. The convex kernel of the set $S$ will be denoted by $K$, and the set of points of local nonconvexity of $S$ will be denoted by $Q$. The boundary operator in $E_{n}$ will be denoted by "bd", "interior" by "int", "closure" by "cl", and "convex hull" by "conv".

Theorem 1 (Valentine [5]). Let $S$ be a closed connected set having

Received by the editors June 18, 1969.

1 This paper comes from a doctoral dissertation with Professor F. A. Valentine at the University of California, Los Angeles, and was supported in part by NSF Grant GP-5279. 
property $P_{3}$ in a topological linear space. Then the set of points of local nonconvexity of $S$ is a subset of the boundary of the convex kernel of $S$.

The proof of the main theorem of this paper is based on the following definition and theorem.

Definition. A set $M$ in $E_{n}$ is said to be a closed m-dimensional manifold if $M$ is a compact connected set, and if for each $\epsilon>0$ each point $x \in M$ is interior to an open set $M(x)$ in $E_{n}$, of diameter less than $\epsilon$, such that $M \cap M(x)$ is homeomorphic to the unit ball in $E_{m}$.

Theorem 2 (VAlentine [5]). Let $S$ be a compact set in $E_{n}$ having property $P_{3}$. If the set $Q$ of points of local nonconvexity if $S$ is contained in the interior of the convex hull of $S$, and if the convex kernel $K$ of $S$ has interior points, then $Q$ can be expressed as a finite union of disjoint closed $(n-2)$-dimensional manifolds.

Main Theorem. The principal result of this paper is the following.

THEOREM. Let $S$ be a compact set in $E_{n}(n \geqq 3)$ having property $P_{3}$. If the set $Q$ of points of local nonconvexity of $S$ is contained in the interior of the convex hull of $S$, and if the convex kernel $K$ of $S$ has interior points, then $S$ can be expressed as the union of two convex sets.

Proof. First we establish the theorem for $E_{3}$. We construct two sets whose union is the set $S$, and show by a series of lemmas that these two sets are convex.

If $S$ satisfies the hypotheses of the Main Theorem, then by Theorem 2, $Q=\bigcup_{i=1}^{m} M_{i}$, where each $M_{i}$ is a closed Jordan curve, and $M_{i} \cap M_{j}=0$ for $i \neq j$. By Theorem 1 , each $M_{i}$ is embedded in bd $K$, and moreover, bd $K$ is topologically equivalent to a 2 -sphere, so by the Jordan Curve Theorem, bd $K-M_{i}=A_{i} \cup B_{i}$ (disjoint), where $\operatorname{cl} A_{i} \cap \operatorname{cl} B_{i}=M_{i}$, for each $i$. If we define $R$ (for red) as the set of all points $x$ of bd $K-Q$ such that the set $\left\{i: x \in A_{i}\right\}$ has an even number of integers in it, and $G$ (for green) as the set of all points $x$ of bd $K-Q$ such that the set $\left\{i: x \in A_{i}\right\}$ has an odd number of integers in it, then bd $K-Q=R \cup G$, and furthermore, $\operatorname{cl} R \cap \mathrm{cl} G=Q$.

Since int $K \neq 0$, we can choose a point $u \in$ int $K$. For any point $t \in E_{3}-K$, ut $\cap$ bd $K$ contains a unique point, which we define to be the projection of the point $t$ onto bd $K$ with respect to $u$.

Lemma 1. The projection of a point $t \in S-K$ onto bd $K$ cannot be a point of local nonconvexity of $S$.

Proof. Since $t \in S$ and $u \in$ int $K$, if $y \in u t$ and $y \neq t$, then $y \in$ int $S$ and hence $y \notin Q$. 
Therefore, if $A$ is the set of all points of $S-K$ which have projections in $R$, and $B$ is the set of all points of $S-K$ which have projections in $G$, Lemma 1 implies that $S=K \cup A \cup B$ (disjoint). It remains only to be shown that $K \cup A$ and $K \cup B$ are convex.

Lemma 2. If $a \in A$ and $b \in B$, then the segment $a b$ contains at least one point either of $K$ or of the complement of $S$.

Proof. Assume, to the contrary, that $a b \subset S-K$. Then as a point moves continuously from $a$ to $b$ along $a b$, its projection onto bd $K$ is defined and moves from $R$ to $G$ in a continuous motion, and hence at least once crosses $Q=\mathrm{cl} R \cap \mathrm{cl} G$, contradicting Lemma 1 .

Lemma 3. If $x$ and $y$ are points of $S$, and $u \in$ int $K$, then the interior of the triangle $\operatorname{conv}(u, x, y)$ contains at most one point of $Q$.

Proof. Assume that $q, q^{\prime} \in \operatorname{int} \operatorname{conv}(u, x, y)$ with $q, q^{\prime} \in Q$, and that $q \neq q^{\prime}$. Then if the segment $q q^{\prime}$ is extended, it will intersect $u x$ or $u y$. Assume then, that for some $t \in u x$, we have $q^{\prime} \in q t$. Since $x \in S$ and $u \in \operatorname{int} K$, it follows that $t \in \operatorname{int} S$, and since $q \in K$, we have $q^{\prime} \in \operatorname{int} S$, which is impossible by the definition of $Q$.

We can complete the proof of the Main Theorem by showing that $K \cup A$ is convex. Let $x, y \in K \cup A$, and consider three cases.

Case 1 . Assume $x, y \in K$. Then $x y \subset K \subset(K \cup A)$.

Case 2. Assume $x \in K$ and $y \in A$. Then $x y \subset S$. If $x y \cap B \neq 0$, then Lemma 2 would imply the existence of a point of $B$ between two points of $K$ on $x y$, which is impossible. So $x y \cap B=0$, and $x y \subset K \cup A$.

Case 3. Assume $x, y \in A$. If $x y$ is not a subset of $K \cup A$, then by use of Lemma 2, $x y$ must contain at least one point of the complement of $S$. Thus by Tietze's Theorem [4, Theorem 4.4. p. 49], the set conv $(u, x, y) \cap S$, being nonconvex, has a point of local nonconvexity, which is also a point of local nonconvexity of $S$, denoted by $q$. Since $x, y \in S$, we have $q \notin x u \cup y u$. Also $q \in x y$ would imply $x y \subset S$. So $q \in$ int $\operatorname{conv}(u, x, y)$. Also, the projections of $x$ and $y$ onto bd $K$ are elements of $R$ (by definition of $A$ ). Since $q \in Q$, we have $q \in \operatorname{cl} G$. Therefore we may choose $z \in G$ close to $q$ and $v \in$ int $K$ close to $u$ so that $x, y, z$ and $v$ are coplanar, with $z \in \operatorname{int} \operatorname{conv}(v, x, y)$, and so that the projections of $x$ and $y$ onto bd $K$ (now with respect to $v$ ) still lie in $R$. Then as a point moves continuously from $x$ to $y$ along $x y$, its projection onto bd $K$ with respect to $v$ begins in $R$, crosses into $G$ (the point $z$ ), and returns to $R$. At these two crossings we can deduce the existence of two different points of $Q$ inside int $\operatorname{conv}(v, x, y)$, violating Lemma 3. So $x y \subset K \cup A$. 
Thus $K \cup A$ is convex, and by symmetry so is $K \cup B$. Hence the Main Theorem is proved for $E_{3}$.

The above proof for $E_{3}$ holds valid for $E_{n}$ with $n>3$. The only change required in the proof is that the $(n-2)$-dimensional manifold $M_{i}$ must be shown to separate bd $K$ (which is topologically equivalent to an $(n-1)$-sphere) into two components $A_{i}$ and $B_{i}$ so that cl $A_{i} \cap \mathrm{cl} B_{i}=M_{i}$. I am very grateful to Professor R. L. Wilder for advising me that this fact follows from his Jordan-Brouwer Type of Separation Theorem for an $n-G C M$ [6, p. 294].

Counterexamples. The necessity of compactness in the Main Theorem is shown by taking $S=A \times E_{1}$, where $A$ is a 5 -pointed star and $E_{1}$ is the real line. The necessity of the condition $Q C$ int conv $S$ is shown by letting $S=A \times[0,1]$. The necessity that the dimension be at least three is shown by the 5 -pointed star in the plane.

\section{REFERENCES}

1. J. M. Marr and W. L. Stamey, A three-point property, Amer. Math. Monthly 69 (1962), 22-25. MR 25 \#489.

2. R. L. McKinney, On unions of two convex sets, Canad. J. Math. 18 (1966), 883886. MR 34 \#1923.

3. F. A. Valentine, A three point convexity property, Pacific J. Math. 7 (1957), 1227-1235. MR 20 \#6071.

4. - Convex sets, McGraw-Hill Series in Higher Math., McGraw-Hill, New York, 1964. MR $30 \# 503$.

5. - The intersection of two convex surfaces and property $P_{3}$, Proc. Amer. Math. Soc. 9 (1958), 47-54. MR 20 \#6072.

6. R. L. Wilder, Topology of manifolds, Amer. Math. Soc. Colloq. Publ., vol. 32, Amer. Math. Soc., Providence, R. I., 1949. MR 10, 614.

California State College, Fullerton, California 92631 